\title{
Intervención Educativa sobre los Conocimientos y Prácticas Referidas a los Riesgos Laborales en Cultivadores de Papa en Boyacá, Colombia
}

\author{
Educational intervention concerning knowledge and practices \\ regarding work-related risks in potato farmers in Boyacá, Colombia
}

\author{
Juan M. Ospina ${ }^{1}$, Fred G. Manrique-Abril ${ }^{2,3}$ y Nelly E. Ariza ${ }^{2}$
}

1 Escuela de Medicina. Facultad de Ciencias de la Salud. Universidad Pedagógica y Tecnológica de Colombia. Tunja.duendex56@yahoo.com

2 Escuela de Enfermería. Facultad de Ciencias de la Salud. Universidad Pedagógica y Tecnológica de Colombia. Tunja.fgma75@hotmail.com, nearjuan@yahoo.com.

3 Facultad de Enfermería. Universidad Nacional de Colombia. Bogotá, Colombia.

Recibido 4 Junio 2008/Enviado para Modificación 13 Diciembre 2008/Aceptado 28 Febrero 2009

\section{RESUMEN}

Objetivo Evaluar El impacto de una intervención educativa realizada sobre los conocimientos, actitudes y prácticas (CAP), que busca modificar comportamientos en las técnicas de aplicación de pesticidas y organofosforados, en una muestra de cultivadores de papa de 7 municipios de la provincia Centro de Boyacá, en relación con la salud ocupacional y los riesgos laborales.

Materiales y métodos Estudio de intervención antes-después, anidado. Un total de 659 cultivadores de papa recibieron una intervención educativa basada en estrategias participativas sobre salud ocupacional y riesgos laborales. Previo y posterior a la intervención se evaluaron los conocimientos, actitudes y prácticas sobre estos tópicos.

Resultados Se registraron cambios estadísticamente significativos en los conocimientos, de menor significación en las actitudes y en las prácticas

Discusión Las intervenciones educativas en poblaciones vulnerables de bajo nivel de escolaridad requieren de acompañamiento y apoyo sostenido en el tiempo para lograr cambios significativos en las prácticas saludables.

Palabras Clave: Salud laboral, educación en salud, promoción de la salud, compuestos organofosforados (fuente: DeCS, BIREME).

\section{ABSTRACT}

Objective Evaluating the impact of educational intervention concerning knowledge, attitudes and practices (KAP) aimed at changing behaviour in how pesticides and organophosphates are applied in a sample of potato farmers from seven municipalities 
in the Boyacá department of Colombia in connection with occupational health and labour risks.

Materials and methods This was a nested, before-after, intervention study. A participative strategy-based occupational health and labour risk educational intervention was used with 659 potato farmers. Knowledge, attitudes and practice concerning these matters were evaluated prior to and following the intervention.

Results Statistically significant changes in knowledge were recorded; lesser significance was recorded regarding attitudes and practices.

Discussion Educational interventions in vulnerable, low scholastic level populations require ongoing accompaniment and support for achieving significant changes in health practice.

Key Words: Occupational health, health education, health promotion, organophosphorus compounds (source: MeSH, NLM).

$\mathrm{S}$ egún la Organización Mundial de la Salud, cada año se intoxican con plaguicidas entre quinientos mil y un millón de personas, de las cuales cinco a veinte mil mueren $(1,2)$. La exposición ocupacional, con mayor riesgo de intoxicación aguda ocurre en agricultores, peones, obreros y exterminadores de plagas (3-5). Para la población hay un peligro latente en las cadenas nutricionales (6).

En Colombia, el uso de plaguicidas genera muchos problemas (7-11). Falta de implementos de protección, hábito laboral y conocimientos erróneos facilitan las intoxicaciones; $85 \%$ de trabajadores usarían overoles y guantes si se los proveyeran y se lavarían las manos si dispusieran de lavabos (12).

Cundinamarca, Boyacá, Nariño y Antioquia, aportan $90 \%$ de la producción de papa. En Boyacá se calculan 35000 familias dependientes del cultivo (13). Labores poco mecanizadas con más mano de obra directa. Promedio de días hombre por hectárea, es 120 , con jornales de $\$ 12000$, diarios a todo costo. El 85 $\%$ del recurso humano proviene de familiares o vecinos (14).

El Ministerio de la Protección Social, La Gobernación de Boyacá y la Universidad Pedagógica y Tecnológica de Colombia convinieron cooperar en el desarrollo de acciones de promoción de salud y prevención de enfermedad, sobre población laboral vulnerable, cultivadores de papa (15).

El estudio se propuso evaluar el impacto de una intervención educativa sobre los conocimientos, actitudes y prácticas (CAP) referidos a salud ocupacional, riesgo laboral, exposición y medidas de protección, en particular al aplicar organofosforados y carbamatos. 


\section{MATERIALES Y METODOS}

Estudio de intervención, con valoración antes-después, sin grupo control. Universo conformado por cultivadores de papa residentes en siete municipios. Por medio del Statcalc de Epi Info $2002^{\circledR}$ se estimó una muestra de 650 participantes para detectar diferencias menores al $4 \%$ con una confiabilidad del $95 \%$ entre los dos momentos de evaluación. Se seleccionaron 659 sujetos.

Luego de valoración mediante encuesta que indagaba sobre CAP en el trabajo, riesgo laboral y protección, hubo una intervención mixta, con autoevaluación, retroinformación, charlas estructuradas y demostraciones prácticas. Posteriormente se aplicó la misma encuesta inicial y se determinó una diferencia estadísticamente significativa en el efecto si en los ítems había porcentajes de cambio superior al $10 \%$. Para valorar el efecto de la intervención se usó la prueba de McNemar (16). El rango de tiempo transcurrido entre las dos evaluaciones fue de 20-30 días.

\section{RESULTADOS}

El 67,5 \% fueron hombres; edad promedio hombres 44,5 años (SD=14,8. Rango $15-82$ ), mujeres 42,3 años ( $\mathrm{SD}=15,5$. Rango $15-79) ; 52,8 \%$ eran mayores de 40 años; 78,5 \% tenñian pareja estable; 92,2 \% sabía leer, 51,3\% había cursado hasta $5^{\circ}$ grado o menos; familia promedio de 4,5 personas $(\mathrm{SD}=2,1) ; 64 \%$ trabajaba para otra persona; el ingreso personal promedio fue de 207617 pesos por mes (US\$ 112,22 aprox.); 92,7 \% estaban afiliados al Sistema General de Seguridad Social (SGSS), de los cuales el 86,1\% en el Régimen Subsidiado; 48,2 $\%$ trabajaba más de 8 horas al día y $24,3 \%$ no tenía día de descanso; $80,5 \%$ no había recibido capacitación en riesgos laborales.

Evaluación del impacto sobre los conocimientos

Se observó impacto mayor al $10 \%$ en el conocimiento de conceptos sobre riesgo laboral: peligros en el trabajo, accidente en el trabajo, enfermedad laboral y posibilidad de perder el trabajo $(\mathrm{p}<0,0001)$. En el reconocimiento de riesgos presentes en el trabajo: humo, gas, vapor, microorganismos, temperatura extrema, movilización de carga y mala relación con compañeros de trabajo $(\mathrm{p}<0,0001)$. Riesgos ergonómicos como posiciones forzadas y uso de herramientas inadecuadas $(\mathrm{p}<0,0001)$. Riesgobiológico como manipulación de excremento animal $(\mathrm{p}<0,0001)$. Riesgos psicosociales: orden público, conflicto con compañero, condiciones incomodas o inseguras, conflicto familiar $(\mathrm{p}<0,0001)$. Utilidad de los elementos de 
protección para evitar accidentes y contacto de sustancias con piel y mucosas $(p<0,0001)$. Se reconoció asociación entre riesgo laboral y síntomas o cuadros clínicos específicos: accidente laboral, enfermedad mental, interrupción del embarazo, malformaciones y hormigueo en manos.

Menor impacto se encontró en otras manifestaciones como agresividad, vómito, zumbido de oídos, anemia, convulsiones y úlcera gástrica (Tabla 1). No se reconoció como riesgo la temperatura extrema, trabajo a la intemperie, olor penetrante, $\mathrm{ni}$ tareas rutinarias o repetitivas $(\mathrm{p}>0,05)$.

Tabla 1. Principales impactos de la intervención sobre los conocimientos en relación con los riesgos laborales y la salud ocupacional

\begin{tabular}{|c|c|c|c|c|c|c|}
\hline Concepto & Item & $\begin{array}{c}\% \\
\text { Previo }\end{array}$ & $\begin{array}{c}\% \\
\text { Posterior }\end{array}$ & $\begin{array}{c}\% \\
\text { Impacto }\end{array}$ & $x^{2}$ & $\mathbf{P}$ \\
\hline \multirow{4}{*}{$\begin{array}{l}\text { Para usted un } \\
\text { riesgo laboral es: }\end{array}$} & $\begin{array}{l}\text { Peligros en el trabajo que } \\
\text { realiza }\end{array}$ & 34,7 & 55,4 & 20,7 & 41,65 & 0,0000 \\
\hline & $\begin{array}{l}\text { Accidentes en el sitio de } \\
\text { trabajo }\end{array}$ & 26,3 & 51,1 & 24,8 & 73,79 & 0,0000 \\
\hline & $\begin{array}{l}\text { Enfermedad relacionada } \\
\text { con el trabajo }\end{array}$ & 35,9 & 48,7 & 12,8 & 21,87 & 0,0000 \\
\hline & $\begin{array}{l}\text { Posibilidad de perder el } \\
\text { trabajo }\end{array}$ & 9,5 & 25,6 & 16,1 & 62,2 & 0,0000 \\
\hline \multirow{2}{*}{$\begin{array}{l}\text { En su trabajo } \\
\text { existen riesgos } \\
\text { como: }\end{array}$} & $\begin{array}{l}\text { Humos gases } \\
\text { Microorganismos }\end{array}$ & $\begin{array}{l}50,0 \\
36,0\end{array}$ & $\begin{array}{l}63,7 \\
56,4\end{array}$ & $\begin{array}{l}13,7 \\
20,4\end{array}$ & $\begin{array}{c}17,05 \\
48,7\end{array}$ & $\begin{array}{l}0,0000 \\
0,0000\end{array}$ \\
\hline & $\begin{array}{l}\text { malas relaciones con sus } \\
\text { compañeros }\end{array}$ & 7,6 & 19,1 & 11,5 & 23,90 & 0,0000 \\
\hline \multirow{4}{*}{$\begin{array}{l}\text { ¿A cuales riesgos } \\
\text { químicos o } \\
\text { biológicos está } \\
\text { expuesto? }\end{array}$} & Aspiración de humo & 34,0 & 49,8 & 15,8 & 24,12 & 0,0000 \\
\hline & Aspiración de gases & 40,3 & 55,1 & 14,8 & 11,55 & 0,0007 \\
\hline & Aspiración de vapores & 28,4 & 45,5 & 17,1 & 30,54 & 0,0000 \\
\hline & Manejo de excrementos & 33,1 & 45,4 & 12,3 & 14,49 & 0,0001 \\
\hline \multirow{3}{*}{$\begin{array}{l}\text { ¿A cuales riesgos } \\
\text { ergonómicos está } \\
\text { expuesto? }\end{array}$} & $\begin{array}{l}\text { Estar inclinado mucho } \\
\text { tiempo }\end{array}$ & 44,4 & 54,2 & 9,8 & 14,14 & 0,0002 \\
\hline & $\begin{array}{l}\text { Cargar peso en la } \\
\text { espalda }\end{array}$ & 49,4 & 59,2 & 9,8 & 17,64 & 0,0000 \\
\hline & $\begin{array}{l}\text { Uso de herramientas } \\
\text { pesadas }\end{array}$ & 26,5 & 42,5 & 16,0 & 57,78 & 0,0000 \\
\hline \multirow{4}{*}{$\begin{array}{c}\text { ¿A cuales riesgos } \\
\text { psicosociales está } \\
\text { expuesto? }\end{array}$} & $\begin{array}{l}\text { Condiciones incomodas o } \\
\text { inseguras de trabajo }\end{array}$ & 22,7 & 40,7 & 18,0 & 39,86 & 0,0000 \\
\hline & $\begin{array}{l}\text { Conflictos con } \\
\text { compañeros }\end{array}$ & 6,6 & 17,0 & 10,4 & 41,97 & 0,0000 \\
\hline & Conflictos con familiares & 8,4 & 18,4 & 10,0 & 24,8 & 0,0000 \\
\hline & $\begin{array}{l}\text { Problemas de orden } \\
\text { publico }\end{array}$ & 1,6 & 11,2 & 9,6 & 44,29 & 0,0000 \\
\hline \multirow{2}{*}{$\begin{array}{l}\text { Los elementos de } \\
\text { protección } \\
\text { personal son }\end{array}$} & $\begin{array}{l}\text { Equipos o dispositivos } \\
\text { para evitar accidentes }\end{array}$ & 27,4 & 49,9 & 22,5 & 57,22 & 0,0000 \\
\hline & $\begin{array}{l}\text { Ropa que evita contacto } \\
\text { de sustancias con la piel }\end{array}$ & 55,8 & 68,4 & 12,6 & 29,82 & 0,0000 \\
\hline \multirow{4}{*}{$\begin{array}{l}\text { Los elementos de } \\
\text { protección sirven } \\
\text { para: }\end{array}$} & $\begin{array}{l}\text { Protegerse de los riesgos } \\
\text { del trabajo }\end{array}$ & 40,0 & 56,4 & 16,4 & 30,39 & 0,0000 \\
\hline & $\begin{array}{l}\text { Evitar que usted se } \\
\text { enferme por su trabajo }\end{array}$ & 48,4 & 60,8 & 12,4 & 19,69 & 0,0000 \\
\hline & $\begin{array}{l}\text { Evitar accidentes en } \\
\text { trabajo }\end{array}$ & 30,1 & 45,2 & 15,1 & 17,83 & 0,0000 \\
\hline & $\begin{array}{l}\text { Trabajar con } \\
\text { incomodidad }\end{array}$ & 35,2 & 16,4 & 18,8 & 17,13 & 0,0000 \\
\hline \multirow{6}{*}{$\begin{array}{l}\text { Lesiones que } \\
\text { pueden causar } \\
\text { los riesgos } \\
\text { presentes en el } \\
\text { trabajo }\end{array}$} & Accidentes de trabajo & 41.3 & 63,6 & 22,3 & 72,08 & 0,0000 \\
\hline & Enfermedad mental & 24,6 & 37,6 & 13,0 & 27,31 & 0,0000 \\
\hline & Interrupción embarazo & 14,4 & 23,7 & 9,3 & 15,75 & 0,0001 \\
\hline & Malformación física & 19,5 & 32,0 & 12,5 & 41,15 & 0,0000 \\
\hline & Convulsiones & 6,8 & 15,3 & 8,5 & 23,02 & 0,0000 \\
\hline & Anemia & 6,2 & 15,5 & 9,3 & 29,07 & 0,0000 \\
\hline
\end{tabular}


En actitudes, cambió el no utilizar elementos de protección por costo y falta de costumbre $(\mathrm{p}<0,0001)$. Apreciable incremento en saber cómo protegerse, lo riesgoso del trabajo y la importancia de afiliarse al SGSS. Impactos más discretos (5-10\%) en la preocupación por uso de pesticidas y riesgos que de ello se derivan, en enfermedad psicológica y accidente laboral (Tabla 2).

Tabla 2. Principales impactos de la intervención sobre las actitudes en relación con los riesgos laborales y la salud ocupacional

\begin{tabular}{|c|c|c|c|c|c|c|}
\hline Concepto & Item & $\begin{array}{c}\% \\
\text { Previo }\end{array}$ & $\begin{array}{c}\% \\
\text { Posterior }\end{array}$ & $\begin{array}{c}\% \\
\text { Impacto }\end{array}$ & $x^{2}$ & $\mathrm{P}$ \\
\hline \multirow{2}{*}{$\begin{array}{l}\text { Razón para no } \\
\text { utilizar elementos } \\
\text { de protección en } \\
\text { el trabajo }\end{array}$} & Son muy costosos & 44,5 & 31,5 & 13,0 & $4,44^{n}$ & 0,0000 \\
\hline & $\begin{array}{l}\text { No acostumbra } \\
\text { usarlos }\end{array}$ & 29,2 & 41,2 & $-12,0$ & $4,19^{*}$ & 0,0000 \\
\hline \multirow{2}{*}{$\begin{array}{l}\text { Noción de peligro } \\
\text { del trabajo }\end{array}$} & $\begin{array}{l}\text { Considera el trabajo } \\
\text { puede ser peligroso }\end{array}$ & 80,5 & 96,2 & 15,7 & $6,26^{*}$ & 0,0000 \\
\hline & $\begin{array}{l}\text { Es importante estar } \\
\text { afiliado a ARS }\end{array}$ & 87,7 & 98,8 & 11,1 & $6,72^{*}$ & 0,0000 \\
\hline \multirow{2}{*}{$\begin{array}{l}\text { Síntomas } \\
\text { percibidos } \\
\text { cuando fumiga }\end{array}$} & Cefalea & 67,7 & 56,8 & 10,9 & 15,65 & 0,0001 \\
\hline & Hormigueo manos & 39,0 & 26,3 & 12,7 & 23,8 & 0,0001 \\
\hline
\end{tabular}

* Por ser tomadas de pregunta de selección múltiple se toman como comparación de porcentajes en muestras independientes, aplica el estadístico Z

No hubo modificación en el riesgo de posturas incorrectas, fortaleza personal, ventajas de utilizar medidas o garantizar la protección laboral.

Impacto sobre las prácticas

Cambios mayores se registraron en: forma de mezclar pesticidas y abonos, tendencia al uso de guantes, tapabocas y overol, baño de manos y cuerpo, y cambio de ropa después de fumigar, almacenamiento de sobrantes, postura para trabajar y recoger la cosecha, uso de gorro para protección del sol.

Cambio menor al $10 \%$ en: uso de gafas de protección y bloqueador solar, posición flexionada para trabajar, consumir agua en el trabajo y tendencia a enterrar las basuras (Tabla 3). Sin cambio en: consumo de alimentos y guarapo al fumigar, periodicidad de fumigación, peso que levanta y carga, almacenamiento de agroquímicos, fuente de obtención del agua para consumo y desechar basuras a campo abierto. Impacto negativo se aprecia en consumir guarapo y chicha durante la jornada $(\mathrm{p}=0,5522)$. 
Tabla 3. Principales impactos de la intervención sobre las prácticas

\begin{tabular}{|c|c|c|c|c|c|c|}
\hline Concepto & Item & $\begin{array}{c}\% \% \\
\text { Previo }\end{array}$ & $\begin{array}{c}\% \% \\
\text { Posterior }\end{array}$ & $\begin{array}{c}\% \% \\
\text { Impacto }\end{array}$ & $x^{2}$ & $P$ \\
\hline \multirow{5}{*}{$\begin{array}{l}\text { Elementos de } \\
\text { protección que } \\
\text { utiliza al trabajar }\end{array}$} & Guantes & 41,2 & 58,1 & 16,9 & 173,8 & 0,0000 \\
\hline & Tapabocas & 31,4 & 47,0 & 15,6 & 29,1 & 0,0000 \\
\hline & Overol & 24,7 & 35,5 & 10,8 & 20,8 & 0,0000 \\
\hline & Gafas & 4,8 & 13,5 & 8,7 & 21,7 & 0,0000 \\
\hline & Sombrero-cachucha & 72,5 & 79,2 & 6,7 & 11,0 & 0,0001 \\
\hline \multirow{3}{*}{$\begin{array}{l}\text { Después de } \\
\text { fumigar }\end{array}$} & Se baña las manos & 31,9 & 43,9 & 12,0 & 19,7 & 0,0000 \\
\hline & Se baña el cuerpo & 59,6 & 69,8 & 10,2 & 24,5 & 0,0000 \\
\hline & Se cambia de ropa & 52,2 & 66,2 & 14,0 & 33,4 & 0,0000 \\
\hline \multicolumn{7}{|l|}{ ¿Qué hace con } \\
\hline $\begin{array}{l}\text { los residuos } \\
\text { cuando sobra en } \\
\text { la fumigación? }\end{array}$ & Los guarda & 45,3 & 60,2 & 14,9 & $5,0^{*}$ & 0,0000 \\
\hline Postura al & De pis & 58,8 & 74,1 & 15,3 & $5,7^{\star}$ & 0,0000 \\
\hline trabajar & Agachado & 25,0 & 19,1 & 5.9 & $2,5^{*}$ & 0,0139 \\
\hline \multirow{2}{*}{$\begin{array}{l}\text { Para recoger la } \\
\text { cosecha }\end{array}$} & Agacha flexionado & 69,4 & 59,3 & 10,1 & $3,4^{*}$ & 0,0005 \\
\hline & Agacha sin flexión & 26,7 & 39,1 & 12,4 & $4,3^{*}$ & 0,0000 \\
\hline \multirow{3}{*}{$\begin{array}{l}\text { Para protegerse } \\
\text { del sol usa }\end{array}$} & Protector solar & 3,7 & 10,9 & 7,2 & 20,8 & 0,0000 \\
\hline & Gorro & 7,7 & 18,4 & 10,7 & 26,4 & 0,0000 \\
\hline & Crema & 6,4 & 11,2 & 4,8 & 6,0 & 0,0145 \\
\hline \multirow{3}{*}{$\begin{array}{l}\text { Se protege del } \\
\text { fría con }\end{array}$} & Guantes & 7,9 & 24,6 & 16,7 & 173,8 & 0,0000 \\
\hline & Saco & 45,3 & 64,2 & 18,9 & 27,5 & 0,0000 \\
\hline & Gorro & 12,3 & 18,4 & 6,1 & 26,4 & 0,0001 \\
\hline \multirow{2}{*}{$\begin{array}{l}\text { Durante la } \\
\text { Jornada } \\
\text { consume }\end{array}$} & Chicha & 2,8 & 7,0 & 4,2 & 12,8 & 0,0003 \\
\hline & Agua & 14,5 & 27,3 & 12,8 & 33,4 & 0,0000 \\
\hline Manejo de las & Las entierra & 2,7 & 13,1 & 10,4 & 39,7 & 0,0000 \\
\hline
\end{tabular}

* Por ser tomadas de pregunta de selección múltiple se toman como comparación de porcentajes en muestras independientes, aplica el estadístico Z.

\section{DISCUSIÓN}

El uso de agroquímicos y fertilizantes en el cultivo de papa, eleva los costos de producción. El agricultor reconoce el daño que causan las plagas y confunde su tipificación, al identificar solo las más tradicionales (17).

Monitorear la exposición a pesticidas es vital (18), por el riesgo en trabajadores que aplican o manipulan a campo abierto o en interiores tales productos; industria alimentaria, trabajadores de salud pública, centros veterinarios, pilotos y los que intervienen en aplicaciones agrícolas (19).

La exposición es intermitente, de intensidad y nivel variables, o simultánea por mezclas. Se puede reducir a límites aceptables, con medidas adecuadas. Las vías inhalatoria y dérmica se relacionan estrechamente con la exposición. La vía digestiva es atípica, pero implica riesgo importante si se consume tabaco, alimentos o bebidas en el trabajo. La absorción por piel no es uniforme para un compuesto. En el caso del parathión $®$, la absorción dérmica varía desde el $0 \%$, en arco plantar, hasta el $100 \%$, en el escroto. (20) 
Aunque hay cambios en el nivel de conocimientos sobre riesgo y protección, ello contrasta con la ausencia de cambio en conceptos y comportamientos muy arraigados en la cultura campesina, que reclaman intervenciones más elaboradas y sostenidas en el tiempo.

En salud pública interesa el efecto de una intervención que modifique el comportamiento social y psicológico en el ámbito laboral (21), En relación con la influencia de los factores sociales en la mente y el comportamiento (22). Enfermedades crónicas, como la intoxicación por organofosforados, de larga duración y curso estable, facilitan la evaluación. Por ello, se han diseñado modelos para medir el impacto de intervenciones psicosociales, aún sobre la influencia del estrés y los aspectos socioeconómicos (23).

Este trabajo evidenció la utilidad de intervenciones educativas que modifiquen actitudes e incorporen comportamientos responsables en los cultivadores como estrategia de salud pública (24).

En la evaluación cuenta la calidad metodológica. Elementos como definición del evento, estandarización de la intervención, sensibilidad y especificidad del instrumento, poder estadístico; tamaño de muestra; sesgos, significación del resultado, importan al interpretar los resultados $(25,26)$.

Idealmente, las intervenciones deben permitir que se alcancen amplios grupos incluyendo estudiantes, familias y maestros (27). Los niños influyen en la actitud y comportamiento de sus padres (28) por lo que las estrategias exitosas en el ámbito escolar deben involucrar a las familias (29).

Importante en el análisis, es la escolaridad y edad de los participantes. Las intervenciones en estas poblaciones afrontan retos como la proporción de analfabetos y personas con bajo nivel instruccional, que chocan con inexperiencia y baja concientización en los trabajadores de la salud (30).

Los enfoques de educación, ubican los procesos de enseñanza aprendizaje en entornos grupales que enfaticen sobre la necesidad de articular el aprendizaje con problemas significativos para adultos y su experiencia inmediata (31).

Las acciones educativas, deben cualificar y dignificar la calidad de vida de estos grupos (32). La capacitación e información brindada a los cultivadores de papa tuvo un efecto positivo en el nivel de conocimientos sobre salud ocupacional y riesgos laborales propios de la actividad que desempeñan 
Agradecimientos: Al Ministerio de la Protección Social, la Gobernación y Secretaría de Salud de Boyacá, grupo de investigación Salud, Trabajo y Calidad de Vida de la UPTC por la financiación y ejecución del programa de promoción de la salud y prevención de la enfermedad dirigido a población vulnerable, cultivadores de papa del Departamento.

\section{REFERENCIAS}

1. García JE. Intoxicaciones agudas con plaguicidas: costos humanos y económicos. Revista Panamericana de Salud Pública 1998;4:383-7.

2. Eddleston M, Karalliedde L, Buckley N, Hutchinson G, Isbister G, Konradsen F et al. Pesticide poisoning in the developing world—a minimum pesticides list. The Lancet 2002;360:1163-7.

3. Bolognesi C, Parrini M, Merlo F, Bonassi S. Frequency of micronuclei in lymphocytes from a group of floriculturists exposed to pesticides. Journal of Toxicology and Environmental Health1993;40(2-3).

4. Joksic G, Vidakovic A, Spasojevic-Tisma V. Cytogenetic Monitoring of Pesticide Sprayers. Environmental Research 1997;75(2):113-118.

5. Falck GCM, Hirvonen A, Scarpato R, Saarikoski ST, Migliore L, Norppa H. Micronuclei in blood lymphocytes and genetic polymorphism for GSTM1, GSTT1 and NAT2 in pesticide-exposed greenhouse workers. Mut. Res. Genetic Toxicology and Environmental Mutagenesis 1999;441(2):225-237.

6. Moutschen J, Moutschen-Dahmen M, Degraeve N. Mutagenicity, carcinogenicity and teratogenicity of insecticides. Mutagenicity, Carcinogenicity and Teratogenicity of Industrial Pollutants (M. Kirsch-Volders, Ed.), Plenum Press, New York 1984:127-203.

7. Silva E, Morales L, Ortiz J. Evaluación epidemiológica de plaguicidas inhibidores de acetilcolinesterasa en Colombia, 1996-1997. Biomedica 2000;20(3):200-9.

8. Toro G, Cardenas O, Varona M. Neurotoxicidad (III parte) Plaguicidas. Acta neurol. colomb 2002;18(1):32-50.

9. Idrovo AJ. Intoxicaciones masivas con plaguicidas en Colombia. Biomedica 1999;19(1):67-76.

10. Keifer M, Rivas F, Moon JD, Checkoway H. Symptoms and cholinesterase activity among rural residents living near cotton fields in Nicaragua. Occupational \& Environmental Medicine 1996;53(11):726-9.

11. Palacios ME, Paz P, Hernández S, Mendoza L. Sintomatología persistente en trabajadores industrialmente expuestos a plaguicidas organofosforados. Salud pública de México 1999;41(1):55-61.

12. Eskenazi B, Marks AR, Bradman A, Harley K, Barr DB, Johnson C, Morga N, Jewell NP. Organophosphate Pesticide Exposure and Neurodevelopment in Young Mexican-American Children. Environmental Health Perspectives 2007;115:792-798.

13. Gobernación de Boyacá. Boletines de prensa N N 106. Tunja: Gobernación Boyacá; 2006.

14. Prieto J. Análisis de Coyuntura del cultivo de la papa en Boyacá. Bogotá: ASOPROPAPA; 2000.

15. Colombia. Ministerio de la Protección Social. Convenio interinstitucional $N^{\circ} 0265$ suscrito entre el Ministerio de la Protección Social, la Gobernación de Boyacá y la Universidad Pedagógica y Tecnológica de Colombia. Bogotá DC: UPTC; 2007.

16. Bangdiwala SI. Evaluación de Programas de Intervención para la Prevención de Accidentes en Ambientes Laborales: Consideraciones Estadísticas. Ciencia \& Trabajo 2006;8(19):31-36.

17. Acero LE. La producción de papa en Boyacá. En: Informe de coyuntura económica regional de Boyacá. Tunja: Banco de la República; 2002. p.46-57.

18. De Araújo AJ, de Lima JS, Moreira JC, Jacob Sdo C, Soares Mde O, Monteiro MC, et al. Multiple exposures to pesticides and impacts on health: a cross-section study of 102 rural workers, Nova Friburgo, Rio de Janeiro State, Brazil. Cien Saude Colet 2007; 12(1):115130. 
19. Obiols J. Plaguicidas organofosforados (I): aspectos generales y toxicocinética.[Consultado: Marzo 27 de 2008]. Disponible en: http:/www.mtas.es/insht/ntp/ntp 512.htm.

20. Durham WF, Wolfe HR. Measurement of the Exposure of Workers to Pesticides. Bull WHO 1962;(26):75-91.

21. Angell M. Disease as a reflection of the psyche [editorial]. N Engl J Med 1985; (312):1570-2.

22. Markovitz JH, Matthews KA, Kannel WB, Cobb JL, D'Agostino RB. Psychological predictors of hypertension in the Framingham study: is there tension in hypertension? JAMA 1993; (270):2439-43.

23. Williams RB, Barefoot JC, Califf RM, Haney TL, Saunders WB, Pryor DB, et al. Prognostic importance of social and economic resources among medically treated patients with angiographically documented coronary artery disease. JAMA 1992;(267):520-4.

24. Liborio M, Tomisani N. Evaluación del impacto estrategias de prevención del Dengue en Rosario. Brazilian Journal of Epidemiology 2004; 7(3):234-3.

25. Ruiz JM. Impacto de las intervenciones psicosociales en las enfermedades crónicas: una mirada crítica a la literatura disponible. Rev Colomb Psiquiat 2007;36(3): 125-36.

26. Bermúdez M, Ruiz JG. Variables y fuentes de error en la medición (confusión, sesgo, azar). En: Ruiz Á, Morillo L, editores. Epidemiología clínica: investigación clínica aplicada. Bogotá: Editorial Médica Internacional; 2004. p. 103-10.

27. Olivares S, Snel J, McGrann M, Glasauer P. Educación en nutrición en las escuelas primarias: realidad actual, necesidades y limitaciones. Food Nutr Agr 1998;(22):57-62.

28. Perry CL, Crokett SJ, Pirie P. Parent involvement with Children's Health Promotions: The Minnesota Home Team. Am J Public Health 1998; (78):1156-1160.

29. Center for Disease Control and Prevention. Guidelines for school health programs to promote lifelong healthy eating. MMWR. 1996;45 (No RR-9):1-41.

30. Piñón-Vegal J, Torres M. Impacto de la promoción y la educación para la salud en los logros de la salud pública cubana. Rev Cubana Med Gen Integr 2001; 17(1):365-85.

31. De Lella C. La técnica de los grupos operativos en la formación del personal docente universitario. Perfiles Educativos. 1978; 1(2):45-51.

32. Kawachi I, Kennedy BP, Lochner K, Prothrow-Stith D. Social capital, income inequality and mortality. Am J Public Health 1997;(87):1491-8. 